\title{
Pillar[5]arene-based 3D hybrid supramolecular polymer for green catalysis in water
}

Yan Cai, ${ }^{\dagger}$ Xin Yan, ${ }^{\dagger}$ Siyuan Wang, Zhiwen Zhu, Moupan Cen, Changjin Ou, Qin Zhao, * Qian Yan, Jin Wang, * and Yong Yao*

School of Chemistry and Chemical Engineering, Nantong University, Nantong, 226019, P.R. China

$\dagger$ These authors contributed equally to this work.

Email:zhao.q@ntu.edu.cn; wangjin107@ntu.edu.cn; yaoyong1986@ntu.edu.cn

\section{Supporting Information (13 pages)}

\begin{tabular}{|l|l|l|}
\hline 1. & Materials and methods & $\mathrm{S} 2$ \\
\hline 2. & Synthesis of pillar[5]arenedimer (DP5) & $\mathrm{S} 3$ \\
\hline 3. & Construction of 3D supra-molecular polymers & $\mathrm{S} 5$ \\
\hline 4. & Application in green catalysis & $\mathrm{S} 6$ \\
\hline
\end{tabular}




\section{Materials and methods}

All reagents were commercially available and used as supplied without further purification. Pillar[5] arene DP5 and guest $\mathbf{G}$ were prepared according to the published procedure. S1,S2,S3 $^{2}$

NMR spectra were recorded on a BRUKER AVANCE III HD 400MHz spectrometer.

Mass spectra were recorded on a Micromass Quattro II triple-quadrupole mass spectrometer using electrospray ionization with a MassLynx operating system.

EDS mapping and Scanning electron microscopy (SEM) investigations were carried out on a Hitachi-S-3400 SEM instrument.

Viscosity was measured by Ubbelohde viscometer.

$\mathrm{UV} / \mathrm{Vis}$ spectra and the optical transmittance were recorded in a quartz cell (light path $10 \mathrm{~mm}$ ) on a ShimadzuUV-3600 spectrophotometer equipped with a PTC-348WI temperature controller.

Transmission electron microscopy (TEM) images were acquired using a Tecnai 20 high-resolution transmission electron microscope operating at an accelerating voltage of $200 \mathrm{keV}$. The sample for high-resolution TEM measurements was prepared by dropping the solution onto a copper grid. The grid was then air-dried.

S1. R. Zhang, C. Wang, R. Long, T. Chen, C. Yan, Y. Yao, Front. Chem., 2019, DOI: 10.3389/fchem.2019.00508.

S2. V. Barba, P. Ramos, D. Jimenez, A. Rivera and A. Meneses, Inorganica Chimica Acta, 2013, 401, 30-37.

S3. C. Li, Chem. Commun., 2014, 50, 12420-12433. 


\section{Synthesis of pillar[5]arenedimer (DP5)}

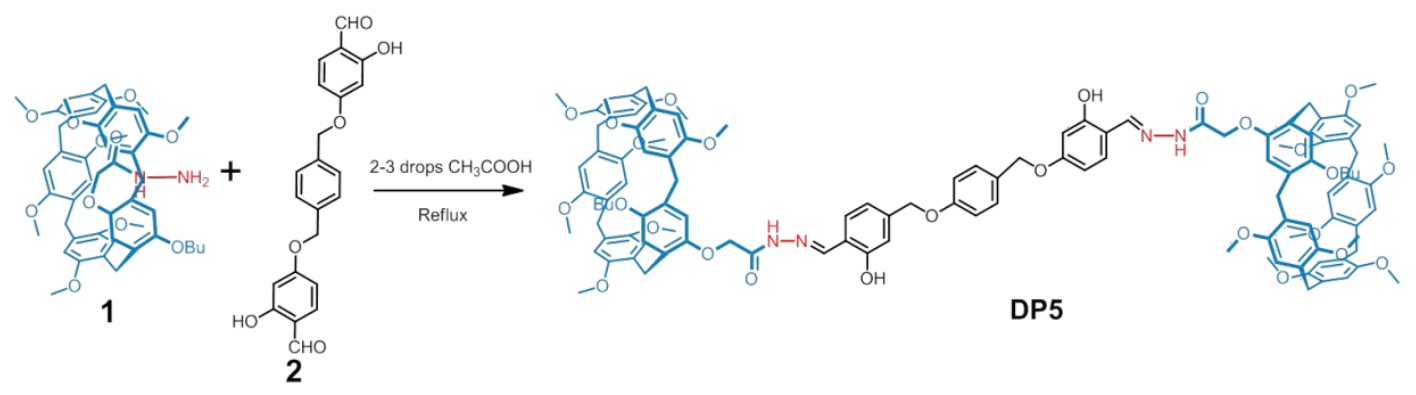

Scheme S1. Synthetic route to pillar[5]areneDP5.

Pillar[5]arene $1(0.17 \mathrm{~g}, 0.20 \mathrm{mmol}), 2(0.04 \mathrm{~g}, 0.10 \mathrm{mmol})$ and $10 \mathrm{~mL}$ $\mathrm{CH}_{3} \mathrm{CH}_{2} \mathrm{OH}$ were added in a $100 \mathrm{~mL}$ flask, then addition of 2-3 drops of $\mathrm{CH} 3 \mathrm{COOH}$, refluxed overnight. The reaction solvent was evaporated and the residue was purified by flash column chromatography on silica gel $\left(\mathrm{CH}_{2} \mathrm{Cl}_{2} / \mathrm{CH}_{3} \mathrm{OH}, v / v 20: 1\right)$ to give DP5 as a white solid $(0.11 \mathrm{~g}$, yield: $55.6 \%)$. m.p. $129.2-131.5{ }^{\circ} \mathrm{C}$; ${ }^{1} \mathrm{H}$ NMR (400 MHz, $\mathrm{CDCl}_{3}$ ) $\delta 11.29$ (s, $\left.2 \mathrm{H}, \mathrm{OH}\right), 9.80$ (s, $2 \mathrm{H}, \mathrm{NH}), 8.26(\mathrm{~s}, 2 \mathrm{H}, \mathrm{CH}=\mathrm{N}), 7.47$ (s, $4 \mathrm{H}, \mathrm{ArH}), 7.06(\mathrm{~d}, J=8.4 \mathrm{~Hz}, 2 \mathrm{H}, \mathrm{ArH})$, $6.80-6.53(\mathrm{~m}, 24 \mathrm{H}, \mathrm{ArH}), 5.10\left(\mathrm{~s}, 4 \mathrm{H}, \mathrm{CH}_{2}\right), 4.46\left(\mathrm{~s}, 4 \mathrm{H}, \mathrm{CH}_{2}\right), 3.88-3.49(\mathrm{~m}$, $\left.66 \mathrm{H}, 48 \mathrm{OCH}_{3}, 18 \mathrm{CH}_{2}\right), 3.32\left(\mathrm{~s}, 6 \mathrm{H}, \mathrm{CH}_{2}\right), 1.76\left(\mathrm{p}, J=7.1 \mathrm{~Hz}, 4 \mathrm{H}, \mathrm{CH}_{2}\right), 1.50$ (q, $\left.J=7.3 \mathrm{~Hz}, 4 \mathrm{H}, \mathrm{CH}_{2}\right), 0.95\left(\mathrm{t}, J=7.4 \mathrm{~Hz}, 6 \mathrm{H}, \mathrm{CH}_{3}\right) ;{ }^{13} \mathrm{C} \mathrm{NMR}(101 \mathrm{MHz}$, $\left.\mathrm{CDCl}_{3}\right) \delta 164.55,161.98,160.62,151.85,151.28,150.90,150.83,150.77,150.68$, $150.64,148.16,136.34,132.17,129.50,129.37,128.76,128.58,128.34,128.11$, $128.08,128.03,127.65,127.47,116.75,115.29,114.95,114.27,114.12,114.04$, $113.75,113.71,113.69,113.49,111.08,107.73,102.45,77.33,69.74,68.61$, $67.76,57.21,56.02,55.99,55.81,55.71,55.68,55.58,31.75,30.21,29.75,29.63$, $29.42,29.05,22.62,19.43,18.76,13.92,11.44$; IR (KBr) v: 3325, 2936, 2833, 1702, 1622, 1503, 1459, 1399, 1210, 1040, 931, 870, 774, 697, 651 cm-1; MS $(\mathrm{m} / \mathrm{z})$ : HRMS (ESI) Calcd. for $\mathrm{C}_{120} \mathrm{H}_{130} \mathrm{~N}_{4} \mathrm{NaO}_{26}{ }^{+}\left([\mathrm{M}+\mathrm{Na}]^{+}\right):$2066.8967, found:2066.8905. 


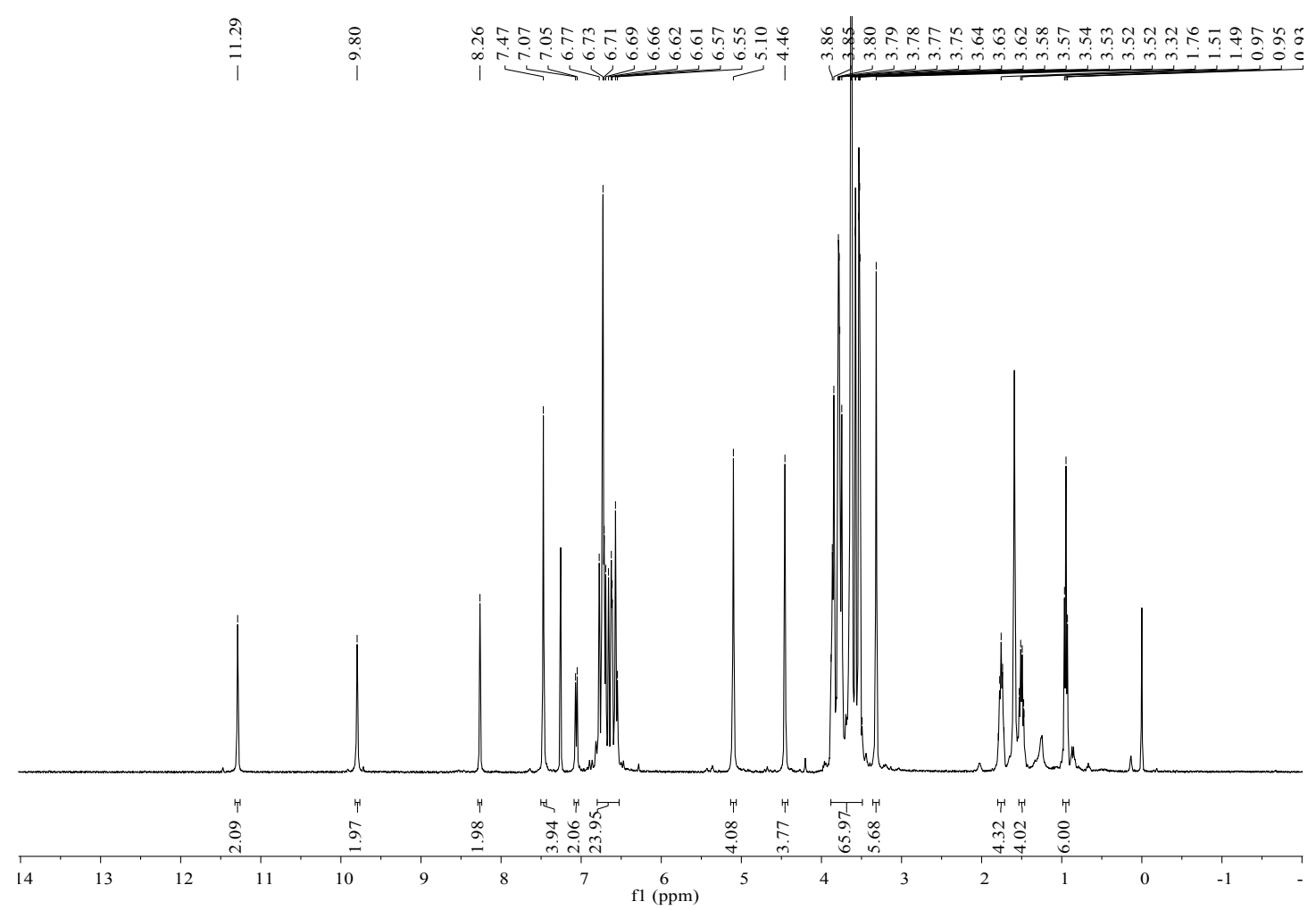

Figure S1. ${ }^{1} \mathrm{H}$ NMR spectra of DP5 in $\mathrm{CDCl}_{3}$ at $400 \mathrm{MHz}$.

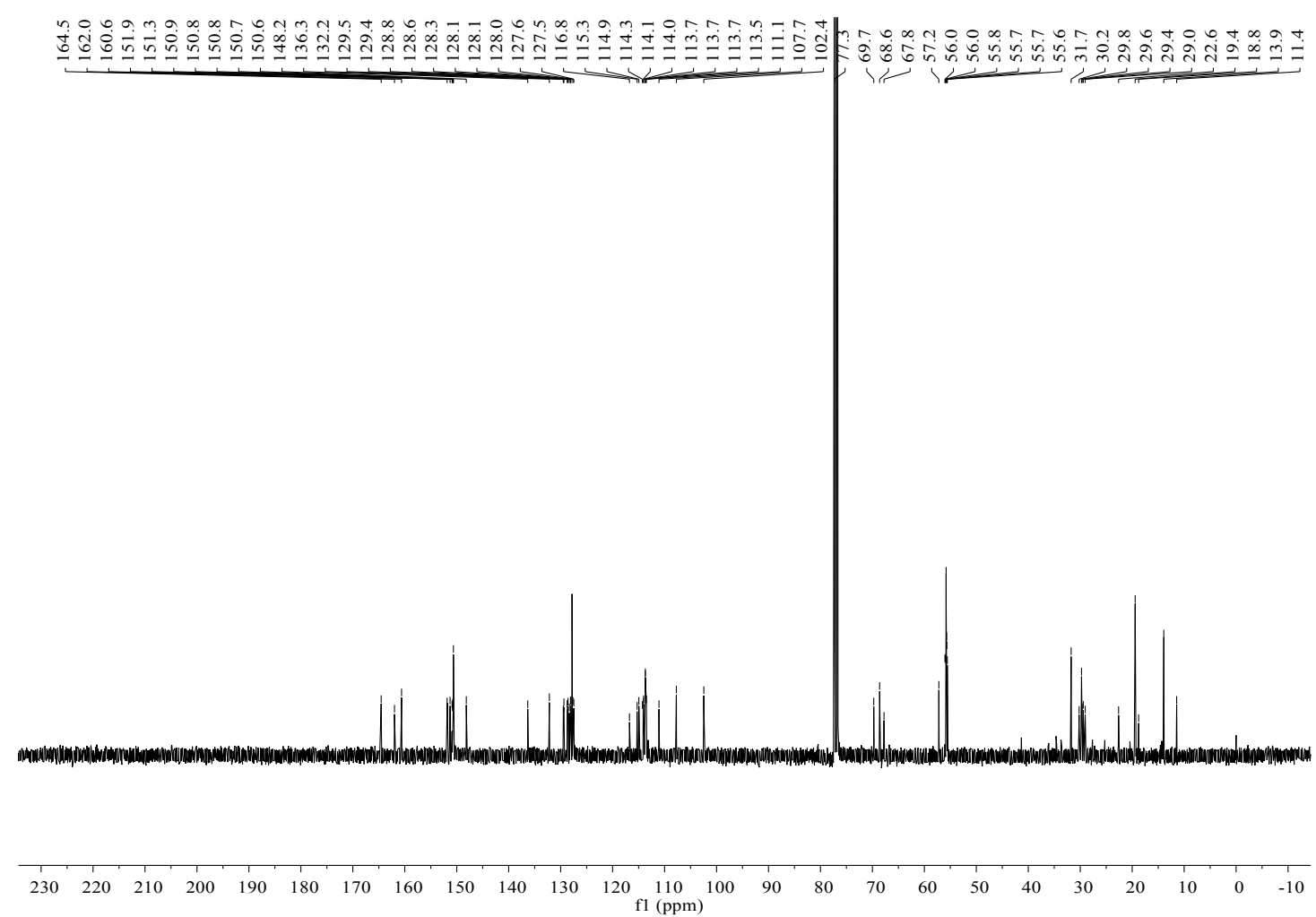

Figure S2. ${ }^{13} \mathrm{C}$ NMR spectra of DP5 in $\mathrm{CDCl}_{3}$ at $100 \mathrm{MHz}$. 


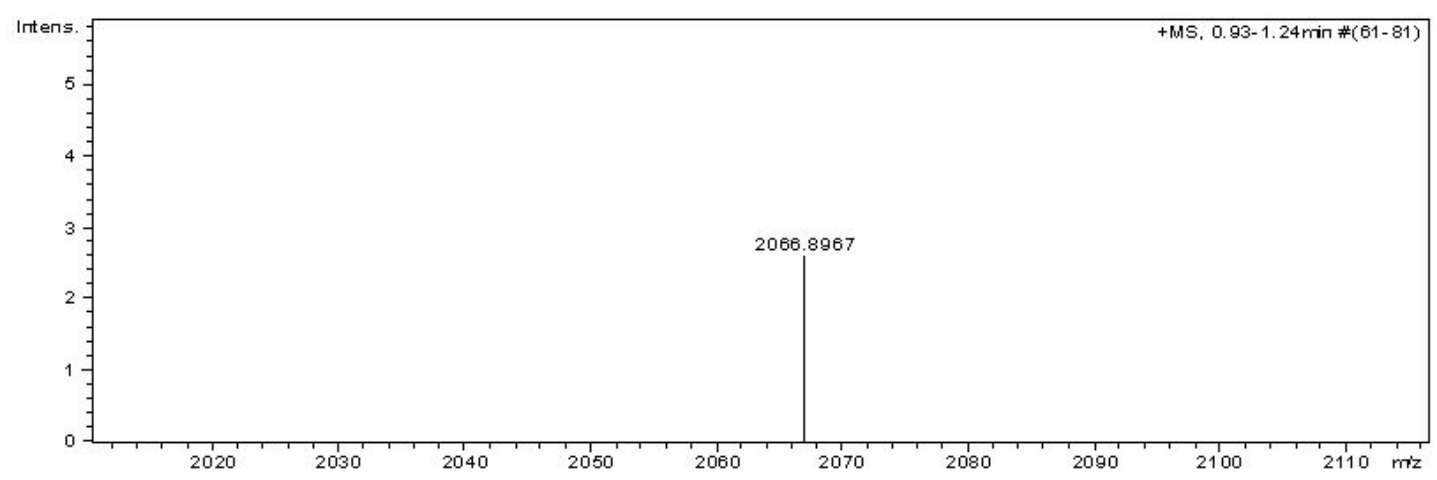

Figure S3. Mass spectra of pillar[5]arene DP5 for $\mathrm{C}_{120} \mathrm{H}_{130} \mathrm{~N}_{4} \mathrm{NaO}_{26}{ }^{+}\left([\mathrm{M}+\mathrm{Na}]^{+}\right)$.

3. Construction of 3D supramolecular polymers

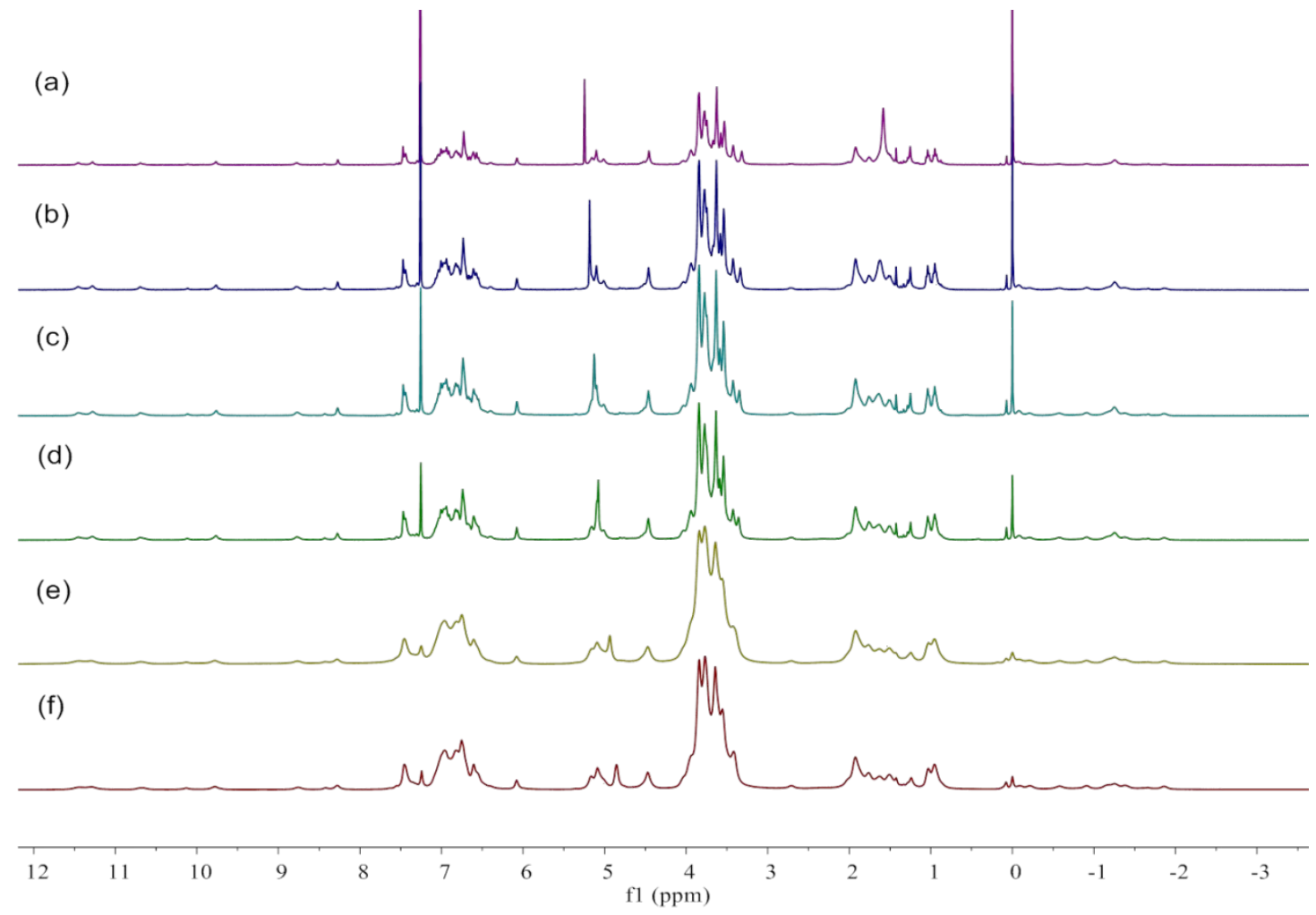

Figure S4. Concentration-dependent ${ }^{1} \mathrm{H}$ NMR spectra (400 MHz, $298 \mathrm{~K}, \mathrm{CDCl}_{3}$ ) of $\mathbf{D P 5} \& \mathbf{G}$ : (a) $3 \mathrm{mmol} / \mathrm{L}$; (b) $8 \mathrm{mmol} / \mathrm{L}$; (c) $12 \mathrm{mmol} / \mathrm{L}$; (d) $20 \mathrm{mmol} / \mathrm{L}$; (e) $40 \mathrm{mmol} / \mathrm{L}$; (f) $50 \mathrm{mmol} / \mathrm{L} .2$ $[\mathbf{D P 5}]=3[\mathbf{G}]$, here the concentration was recorded by [DP5]. 


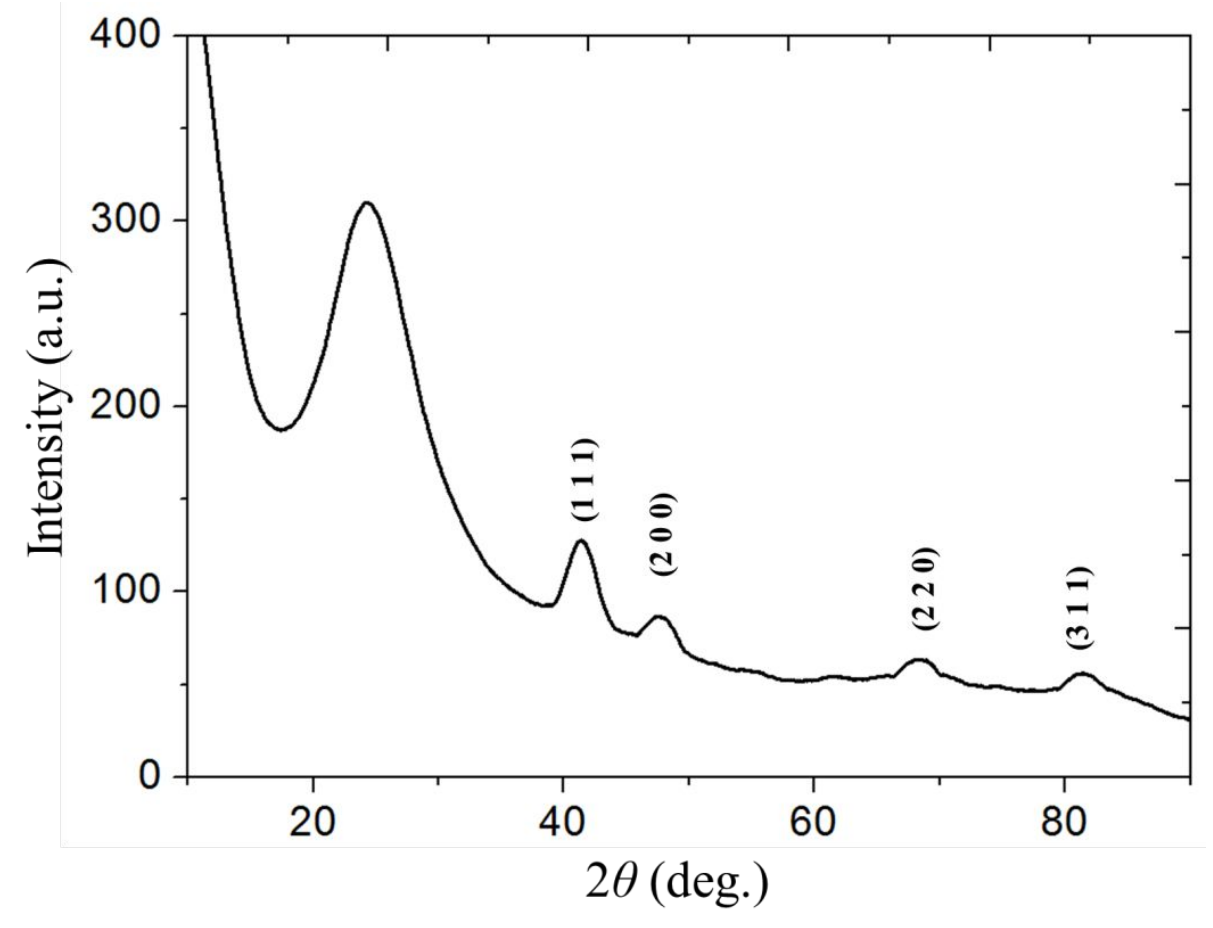

Figure S5. XRD patterns of hybrid 3D supramolecular polymer.

4. Application in green catalysis

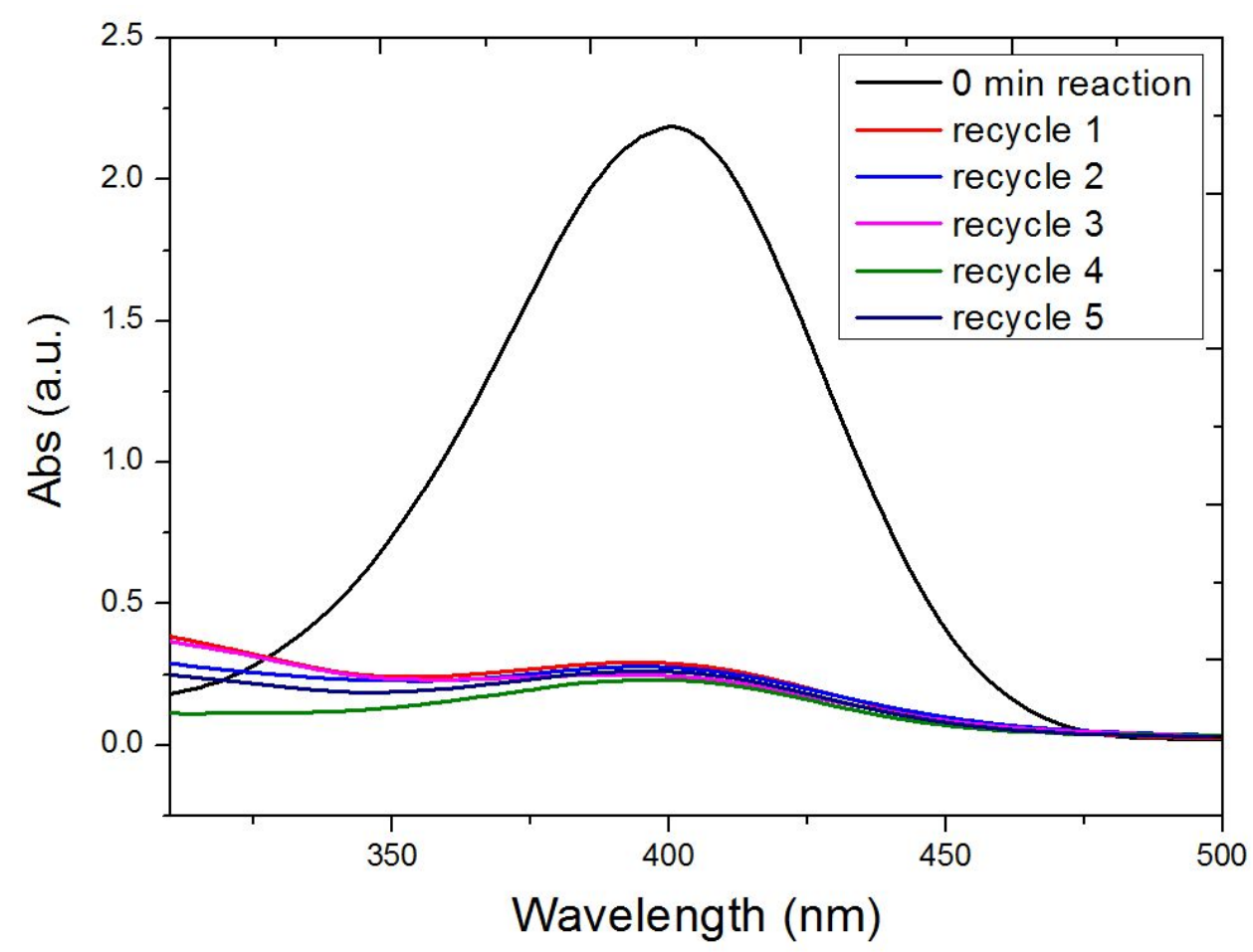

Figure S6. UV-Visible spectra of the catalytic reduction reusing Pd hybrid polymer. $P$-nitrobenzene $(0.02 \mathrm{mM}, 3 \mathrm{~mL})$ was reduced in the presence of $\mathrm{NaBH}_{4}(0.20 \mathrm{mmol})$ by hybrid polymer $(0.10 \mathrm{mg})$. 

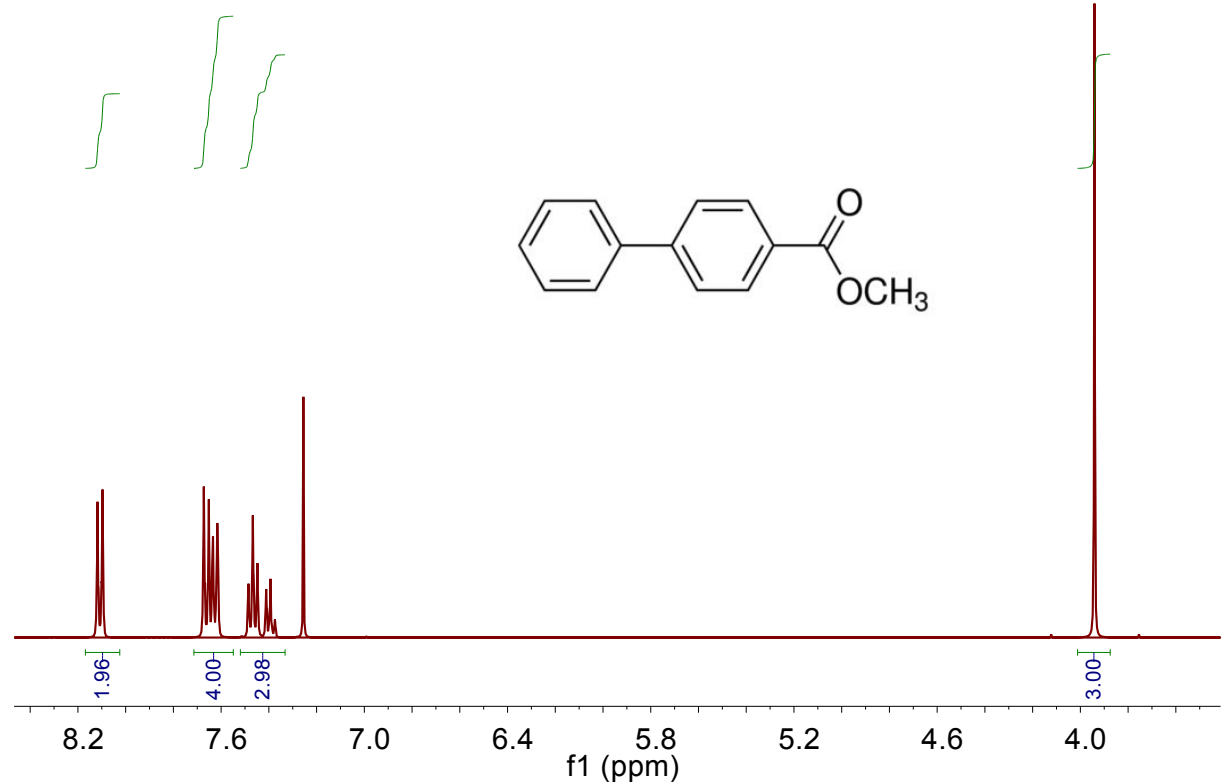

Figure S7. ${ }^{1} \mathrm{H}$ NMR spectra of Methyl 4-biphenylcarboxylate in $\mathrm{CDCl}_{3}$ at $400 \mathrm{MHz}$.

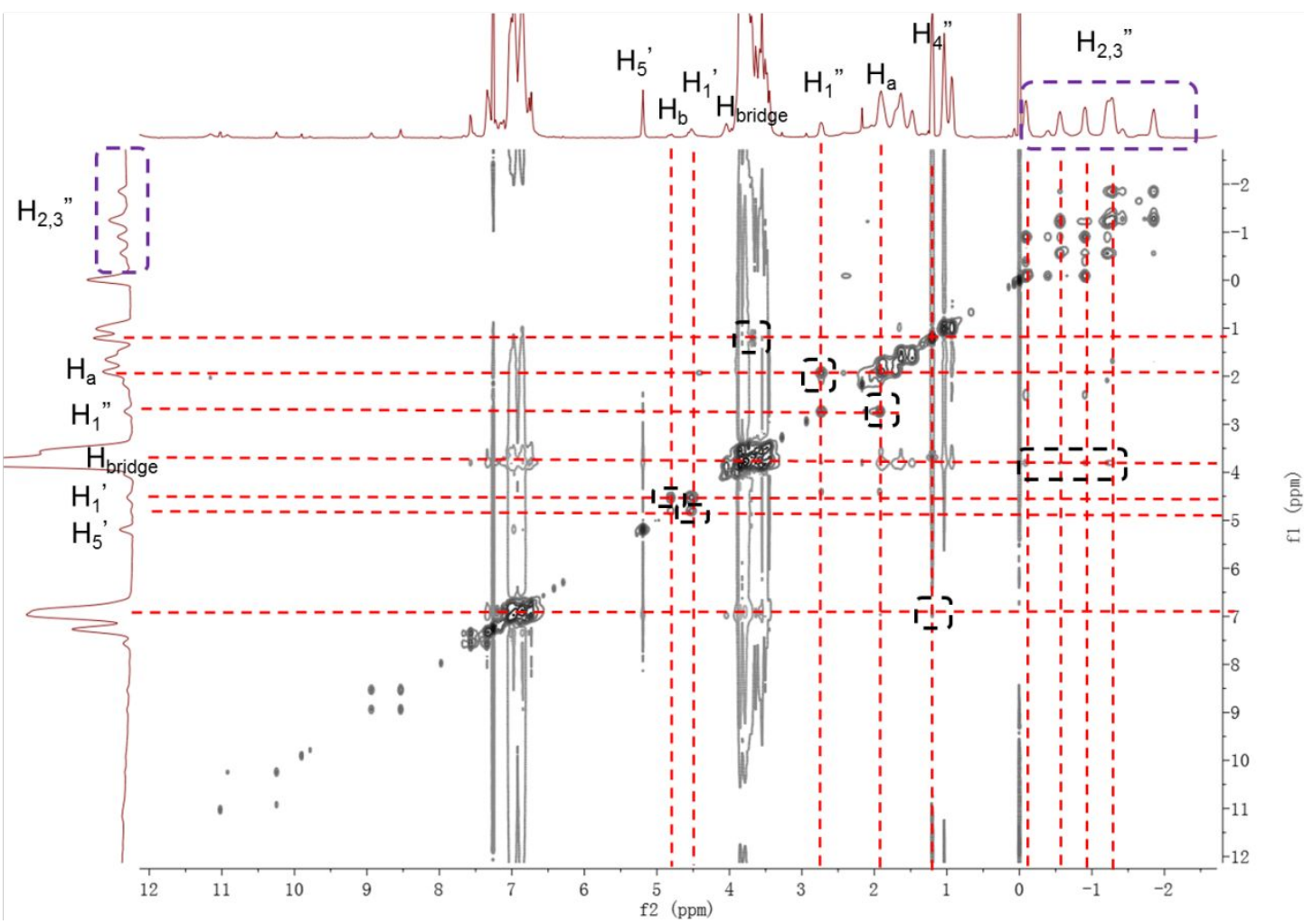

Figure S8. NOE spectrum of $\mathbf{P 5}$ and $\mathbf{G m}$. 


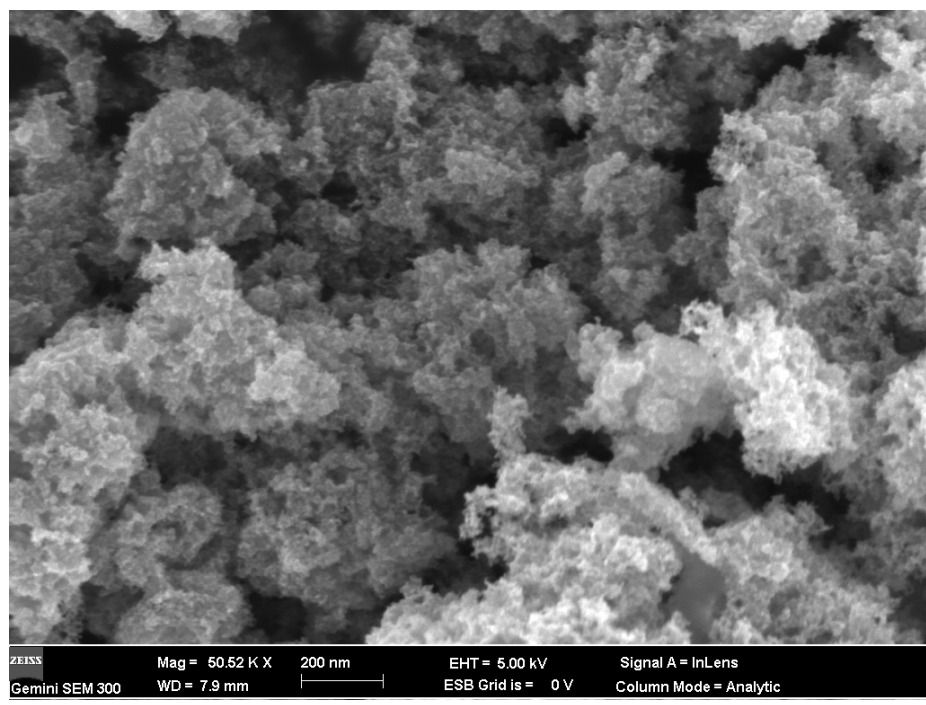

Figure S9. SEM image of the hybrid polymer after usage.

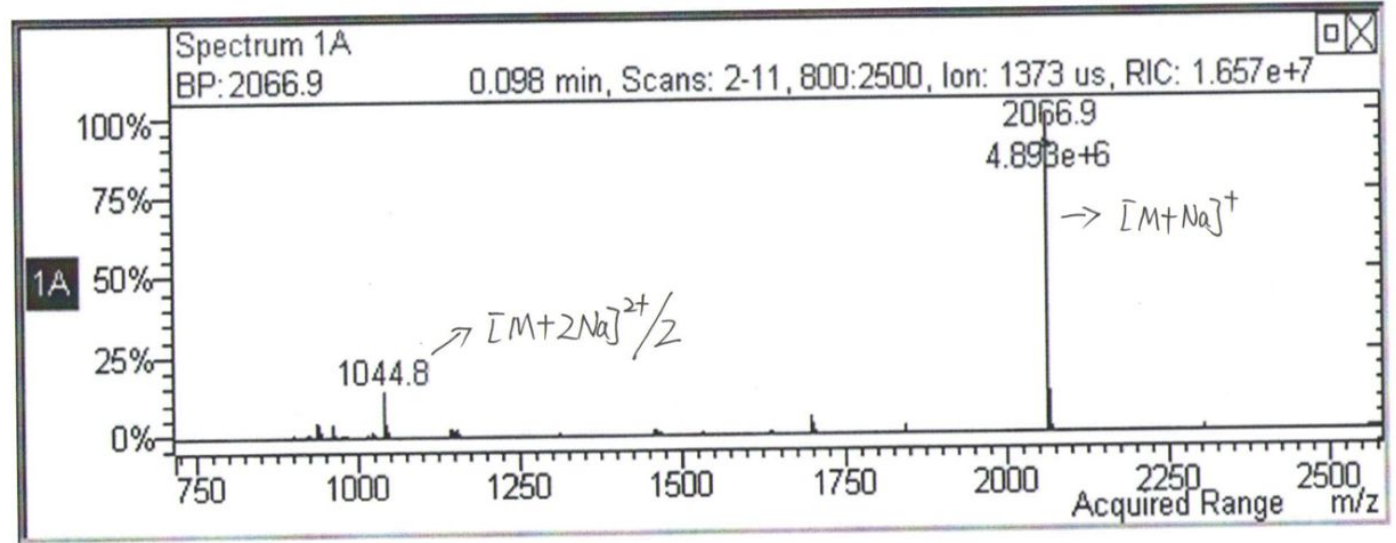

Figure S10. Mass spectra of pillar[5]arene DP5.

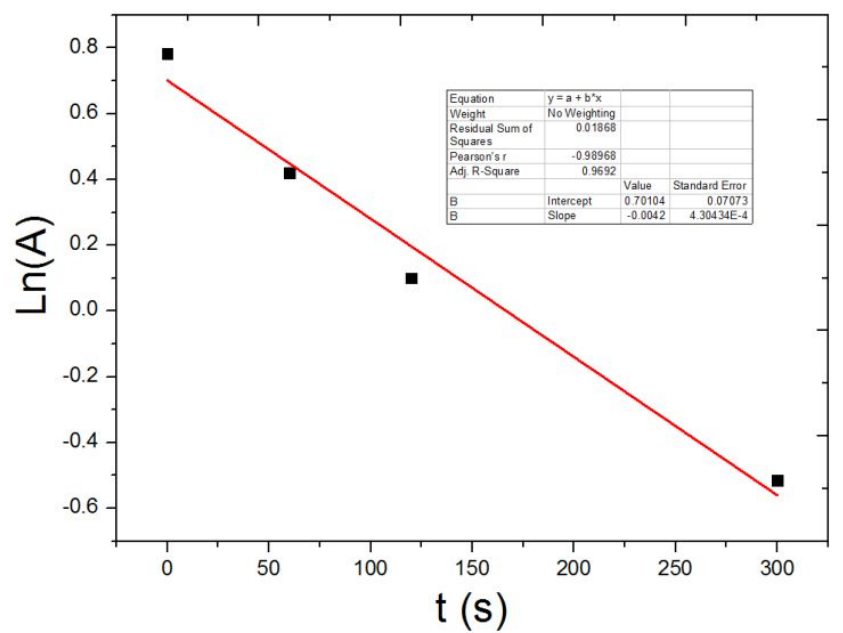

Fig. S11. plot of $\ln (\mathrm{A})$ against time by excess $\mathrm{NaBH} 4$ in the presence of hybrid supramolecular polymers. 


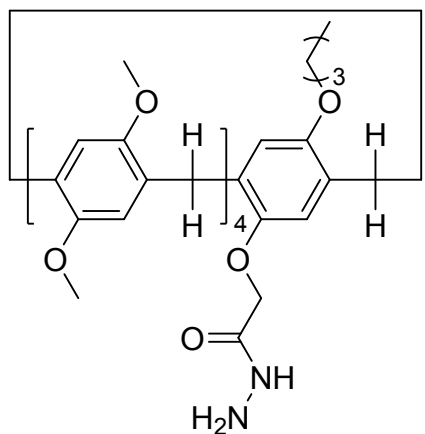

1
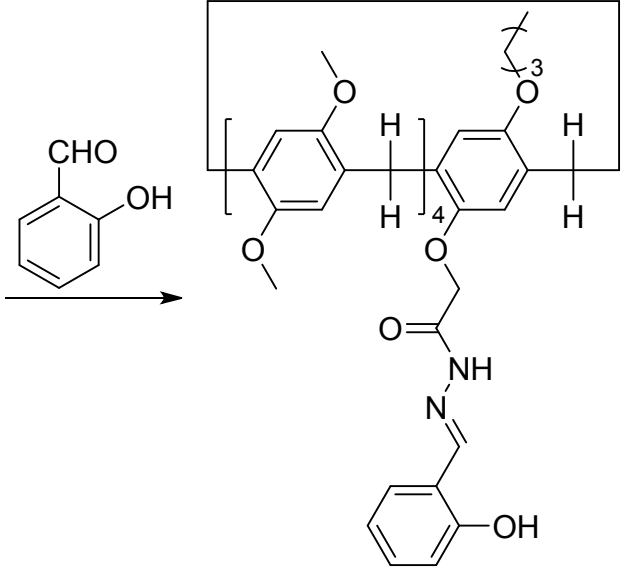

P5

1 (855 mg, $1 \mathrm{mmol})$, salicylaldehyde (122 mg, $1 \mathrm{mmol})$ and acetic acid (2-3d) were refluxed in ethanol $(20 \mathrm{~mL})$ for 12 hours. After cooling, the resulting precipitate was collected by filtration and washed with cold ethanol to give the white solid P5.

P5: White solid, $83.6 \%{ }^{1} \mathrm{H}$ NMR $\left(400 \mathrm{MHz}, \mathrm{CDCl}_{3}\right) \delta 9.93(\mathrm{~s}, 1 \mathrm{H}, \mathrm{NH}), 8.35(\mathrm{~s}, 1 \mathrm{H}$, $\mathrm{OH})$, 7.36-7.32 (m, 1H, ArH), 7.19-7.17 (m, 1H, ArH), 7.04-7.00 (m, 1H, ArH), 6.96-6.92 (m, 1H, ArH), 6.78-6.58 (m, 10H, ArH), $4.48\left(\mathrm{~s}, 2 \mathrm{H}, \mathrm{CH}_{2}\right), 3.89-3.51(\mathrm{~m}$, $\left.34 \mathrm{H}, \mathrm{CH}_{2}, \mathrm{OCH}_{3}\right), 3.32\left(\mathrm{~s}, 2 \mathrm{H}, \mathrm{CH}_{2}\right), 1.80-1.73\left(\mathrm{~m}, 2 \mathrm{H}, \mathrm{CH}_{2}\right), 1.55-1.50(\mathrm{~m}, 2 \mathrm{H}$, $\mathrm{CH}_{2}$ ), 1.09-1.05 (m, 2H, $\left.\mathrm{CH}_{2}\right), 0.95$ (t, $\left.J=7.4 \mathrm{~Hz}, 3 \mathrm{H}, \mathrm{CH}_{3}\right)$.

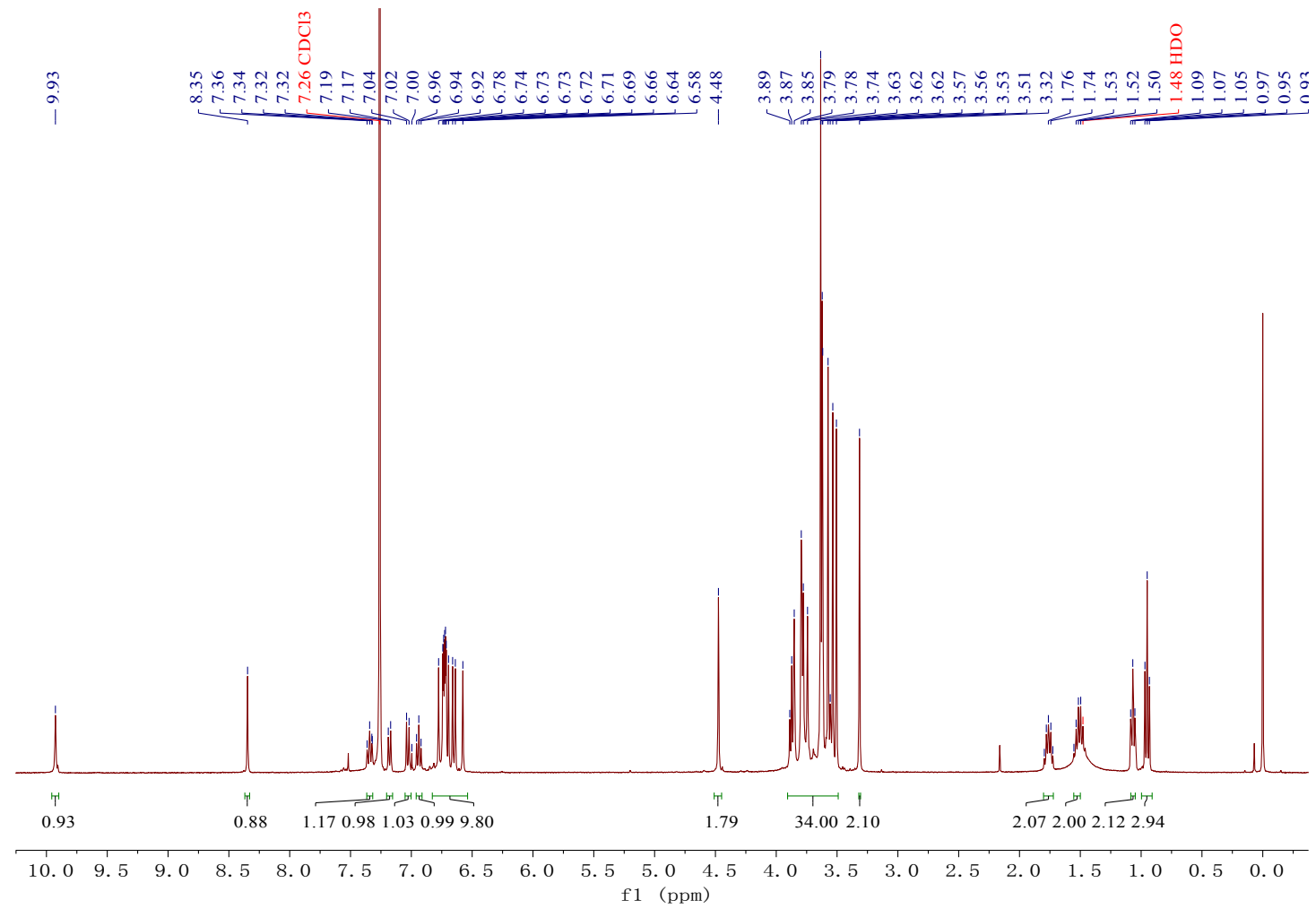

Fig. S12 ${ }^{1} \mathrm{H}$ NMR spectra of $\mathbf{P 5}$ in $\mathrm{CDCl}_{3}$ at $400 \mathrm{MHz}$. 


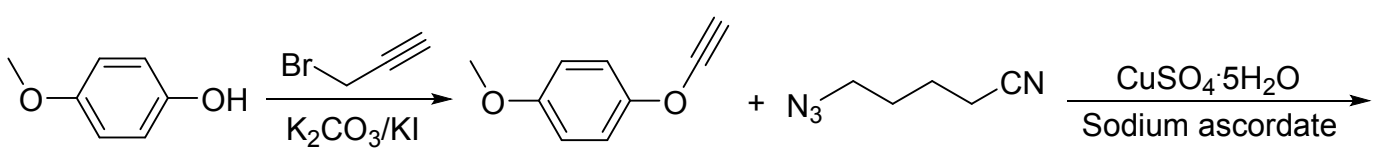<smiles>COc1ccc(OCc2cn(CCCCC#N)nn2)cc1</smiles>

$\mathbf{G}_{\mathbf{m}}$

Methoxy-4-(prop-2-ynyloxy)benzene $(0.38 \mathrm{~g}, 2.3 \mathrm{mmol})$ and 5 azidepenpanenitrile $(0.31 \mathrm{~g}, 2.34 \mathrm{mmol})$ were dissolved in DMF $(10 \mathrm{~mL})$, then sodium ascorbate $(0.20 \mathrm{~g})$ and $\mathrm{CuSO}_{4}(0.16 \mathrm{~g})$ were added. The mixture was stirred at $90{ }^{\circ} \mathrm{C}$ for $14 \mathrm{~h}$. Then, the mixture was poured into brine $(30 \mathrm{~mL})$ and extracted with $\mathrm{CH}_{2} \mathrm{Cl}_{2}(2.30 \mathrm{~mL})$, the organic phase was dried overnight with anhydrous $\mathrm{Na}_{2} \mathrm{SO}_{4}$. The organic phase was concentrated under vacuum and subjected to silica gel chromatography (silica gel, PE $: \mathrm{EA}=1: 1)$ to give the white product $\mathbf{G}_{\mathbf{m}}$.

$\mathbf{G}_{\mathbf{m}}$ : White solid, $58 \% .{ }^{1} \mathrm{H}$ NMR (400 MHz, CDCl3) $\delta(\mathrm{ppm}): 7.61$ (s, 1H, ArH), 6.95-6.91 (m, 2H, ArH), 6.86-6.82 (m, 2H, ArH), $5.17\left(\mathrm{~s}, 2 \mathrm{H}, \mathrm{OCH}_{2}\right), 4.43$ (t, $J=6.8$ $\left.\mathrm{Hz}, 2 \mathrm{H}, \mathrm{NCH}_{2}\right), 3.77$ (s, 3H, $\left.\mathrm{OCH}_{3}\right), 2.41\left(\mathrm{t}, J=7.0 \mathrm{~Hz}, 2 \mathrm{H}, \mathrm{CH}_{2}\right), 2.14-2.05$ (m, 2H, $\left.\mathrm{CH}_{2}\right), 1.73-1.65\left(\mathrm{~m}, 2 \mathrm{H}, \mathrm{CH}_{2}\right)$.
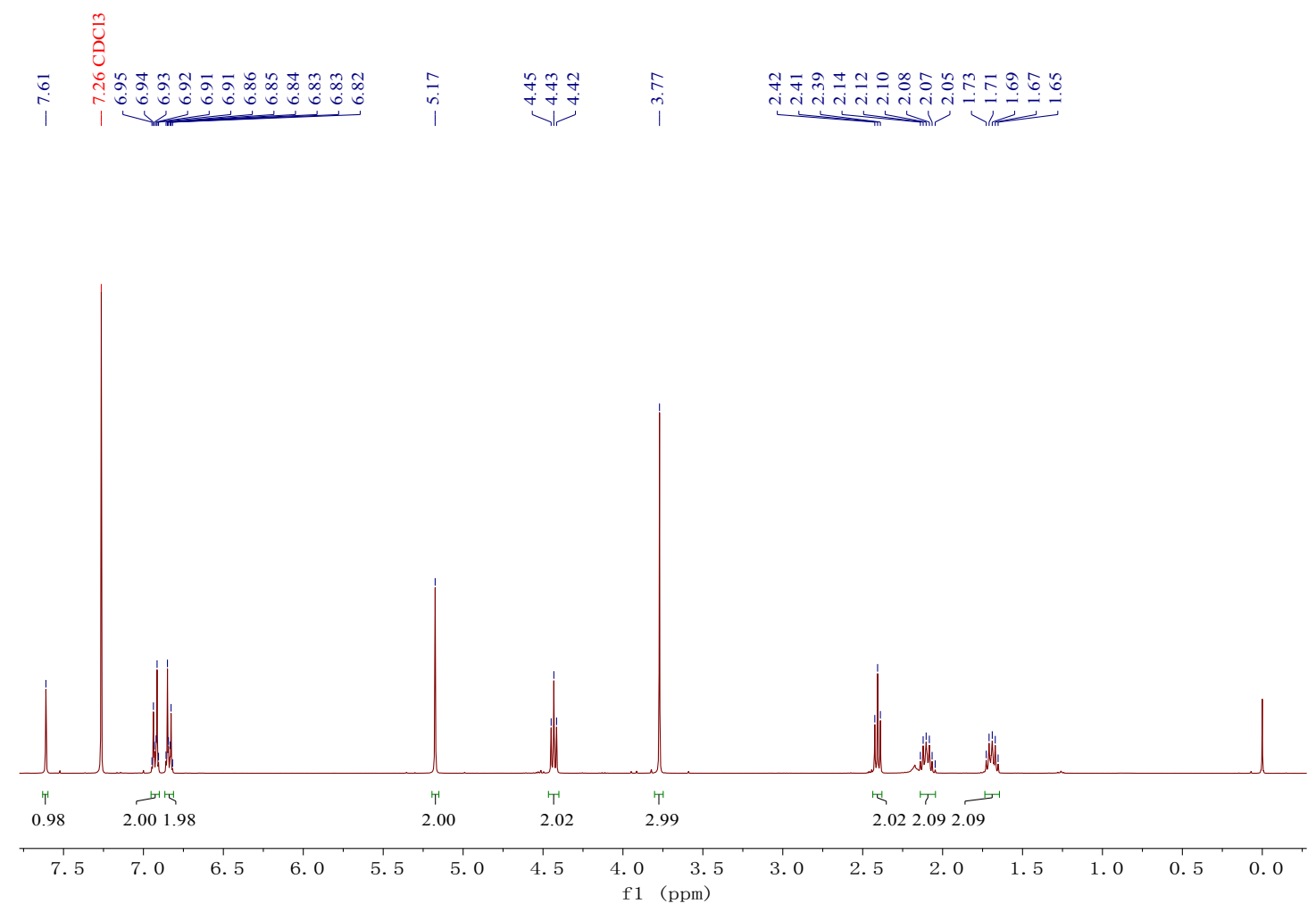

Fig. S13. ${ }^{1} \mathrm{H}$ NMR spectra of $\mathbf{~} \mathbf{m}$ in $\mathrm{CDCl}_{3}$ at $400 \mathrm{MHz}$. 

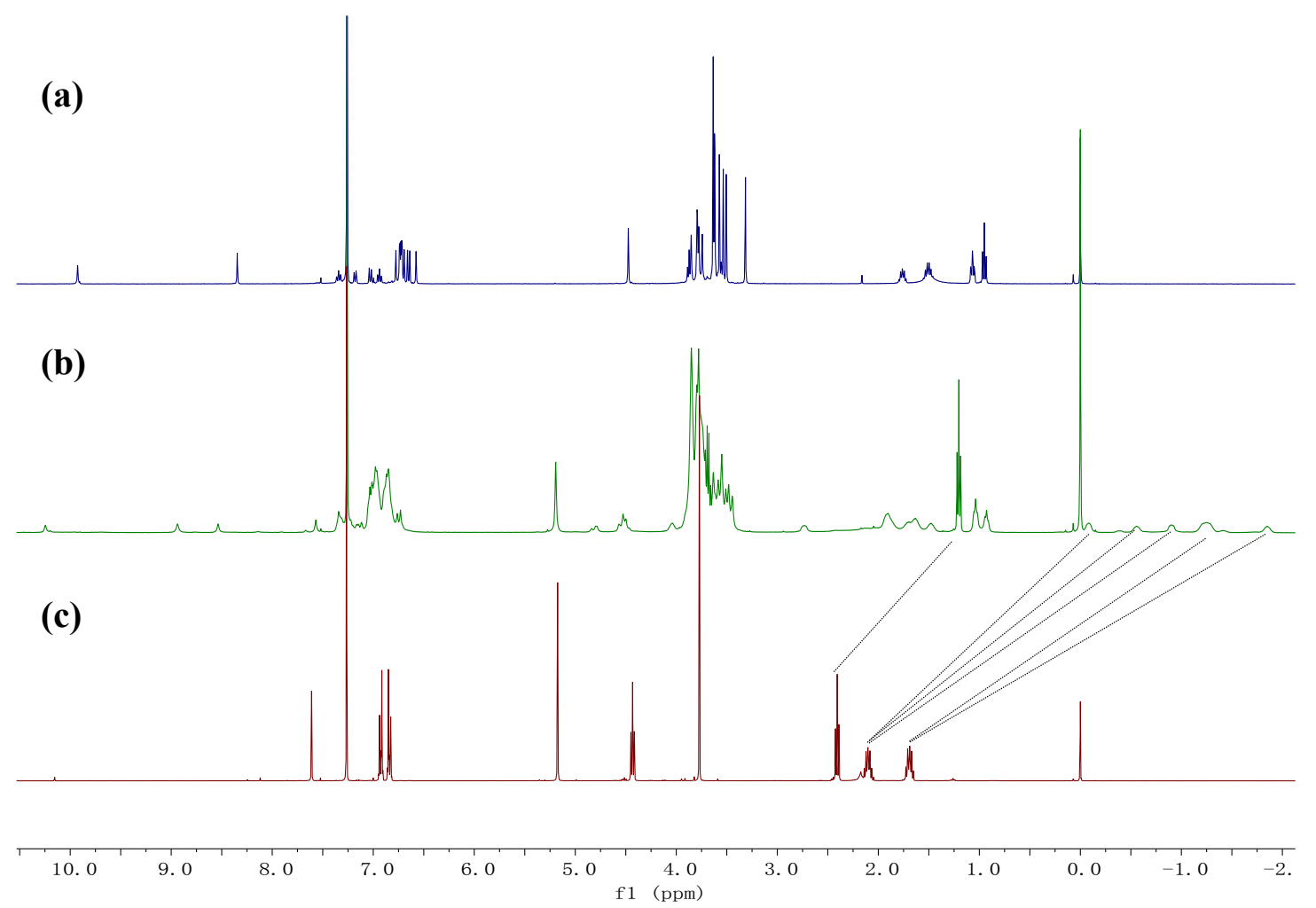

Fig. S14. ${ }^{1} \mathrm{H}$ NMR spectra (400 MHz, $\mathrm{CDCl}_{3}, 298 \mathrm{~K}$ ) of (a) P5 (10.0 mM), (b) $\mathbf{P 5}+\mathbf{G}_{\mathbf{m}}\left([\mathbf{P 5}]=10.0 \mathrm{mM},\left[\mathbf{G}_{\mathbf{m}}\right]=10.0 \mathrm{mM}\right)$, and $(\mathrm{c}) \mathbf{G}_{\mathbf{m}}(10.0 \mathrm{mM})$.
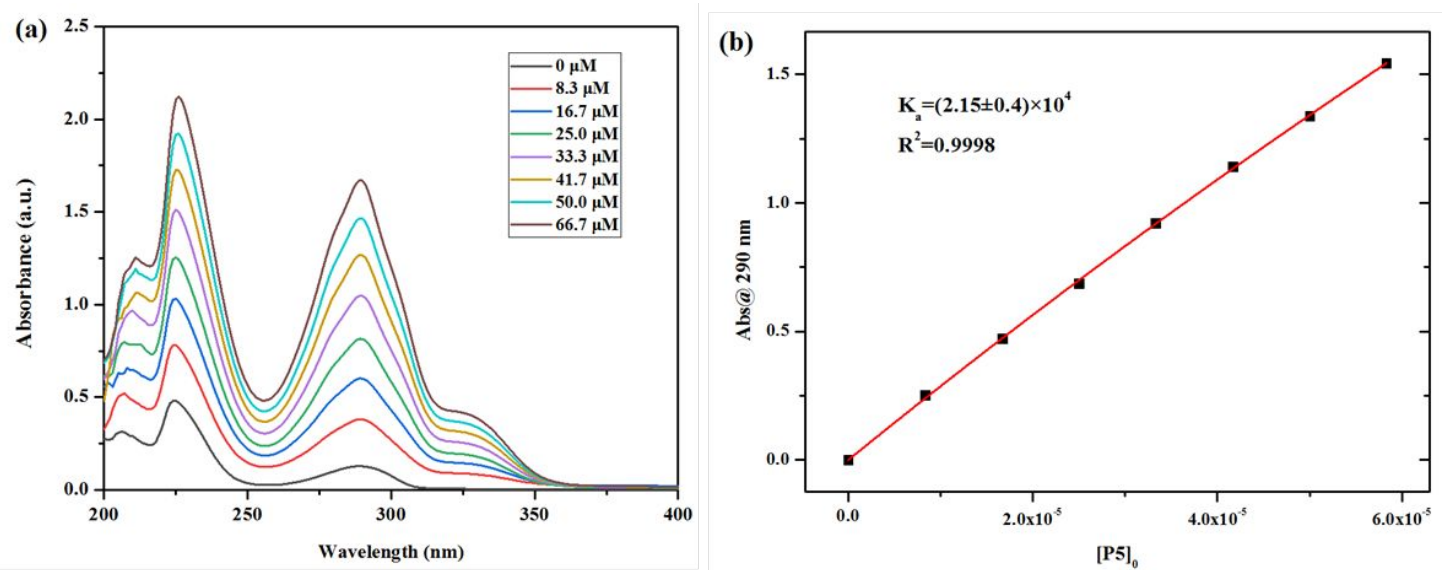

Fig. S15. a) UV-Vis absorption spectra of $\mathbf{G}_{\mathbf{m}}$ at a constant concentration of $50.0 \mu \mathrm{M}$ with different concentrations of $\mathbf{H}_{\mathbf{m}}$. b) The absorption changes of Ha on $\mathbf{G}_{\mathbf{m}}$ upon the addition of $\mathbf{H}_{\mathbf{m}}$. The black solid line was obtained from the non-linear curve-fitting using eq. S1. The association constant of $\mathbf{H}_{\mathbf{m}}$ and $\mathbf{G}_{\mathbf{m}}$ was estimated to be $(2.15 \pm 0.4) \times$ $10^{4} \mathrm{M}^{-1}$.

$\Delta \delta=\left(\Delta \mathrm{A} /[\mathrm{G}]_{0}\right)\left(0.5[\mathrm{H}]_{0}+0.5\left([\mathrm{G}]_{0}+1 / K_{a}\right)-\left(0.5\left([\mathrm{H}]_{0}^{2}+\left(2[\mathrm{H}]_{0}\left(1 / K_{a}-[\mathrm{G}]_{0}\right)\right)+\left(1 / K_{a}\right.\right.\right.\right.$ $\left.\left.\left.\left.+[\mathrm{G}]_{0}\right)^{2}\right)^{0.5}\right)\right) \quad($ eq.S1) 


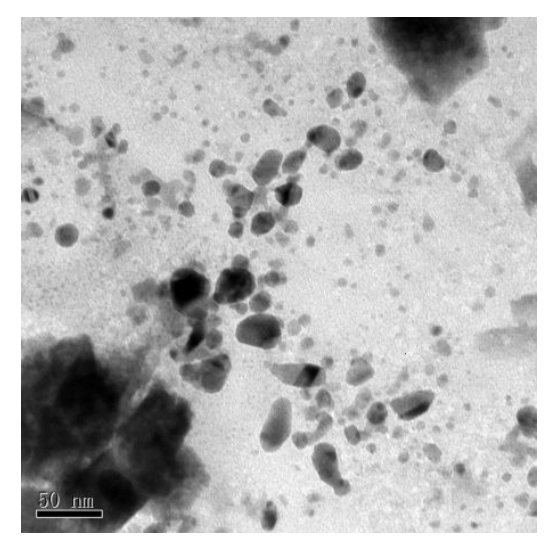

Fig. S16. TEM image of DP5 modified Pd nanoparticles.

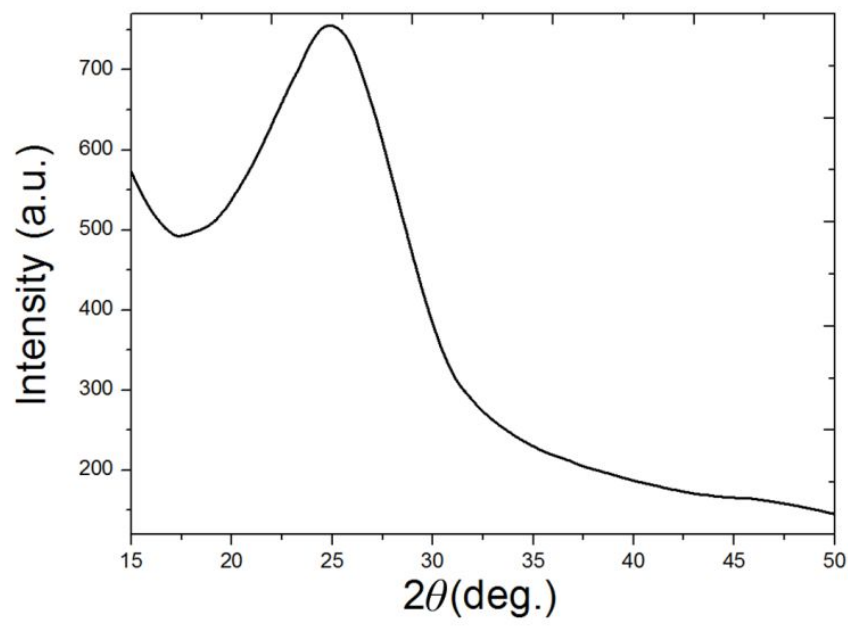

Figure S17. XRD patterns of pure DP5 $\supset$ G supramolecular polymer.

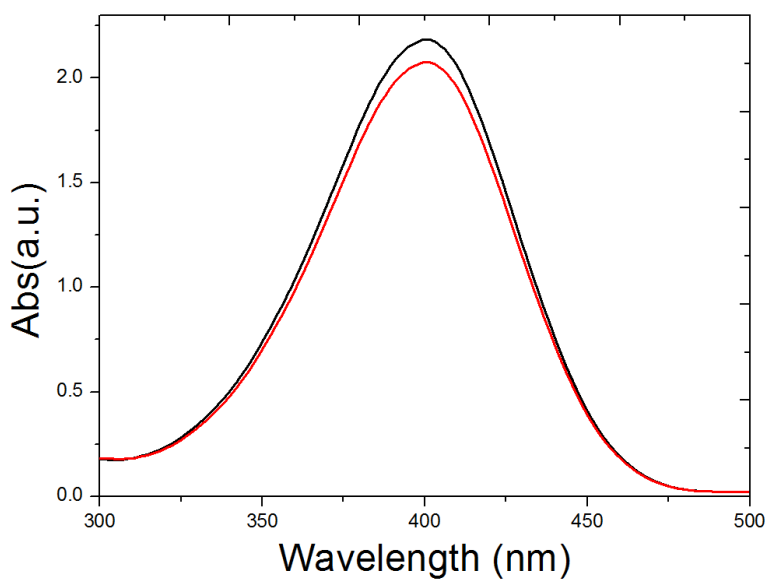

Fig. S18. UV-Visible spectra of the reduction of $P$-nitrophenol $(0.02 \mathrm{mM}, 3 \mathrm{~mL})$ in the presence of $\mathrm{NaBH}_{4}(0.20 \mathrm{mmol})$ after $24 \mathrm{~h}$. 

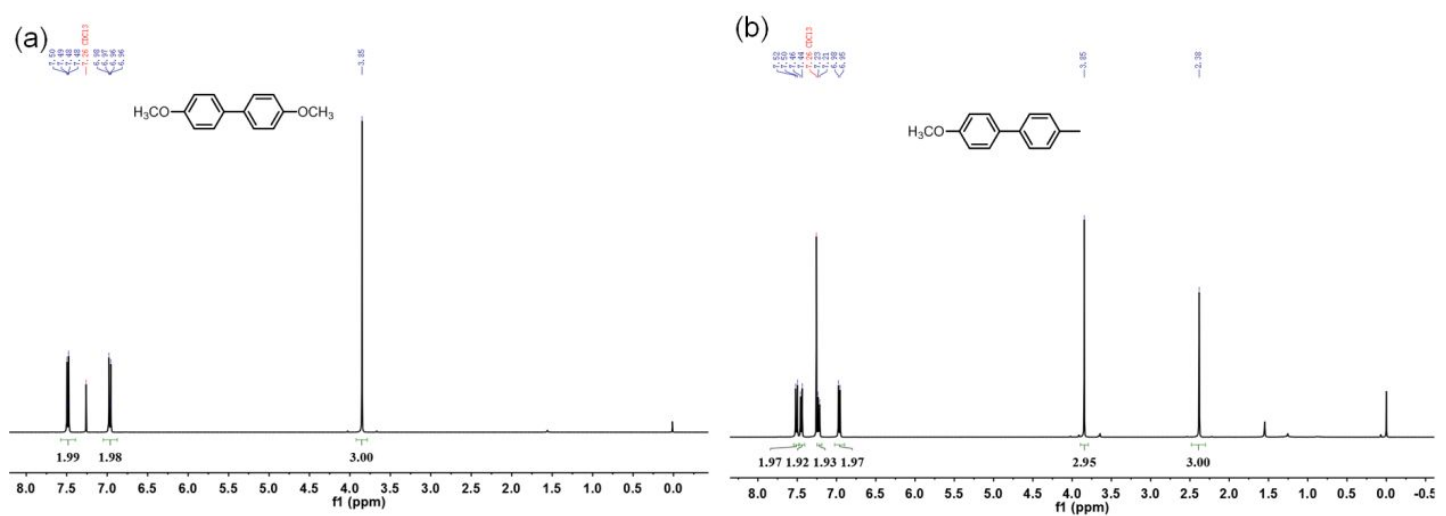

(c)

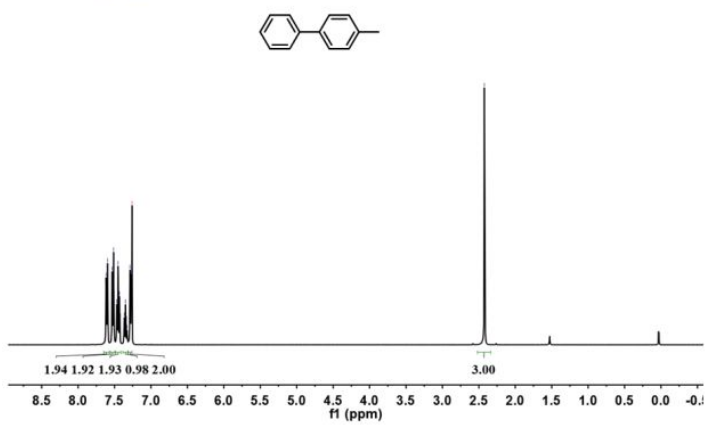

(d)

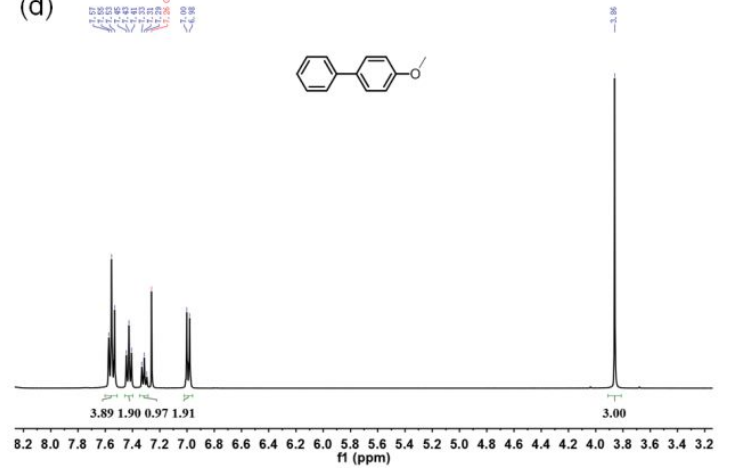

(f)

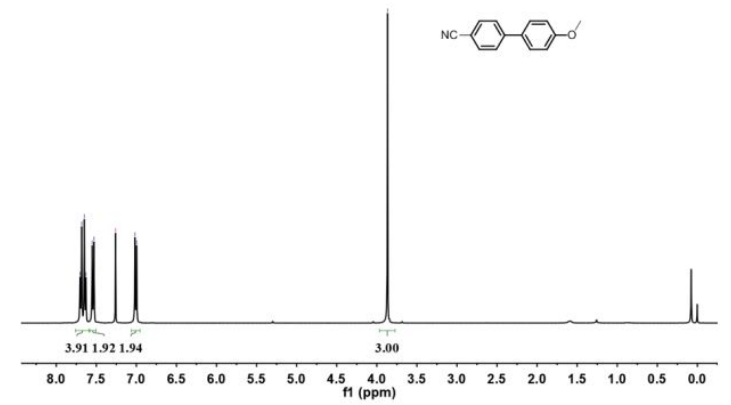

(g)
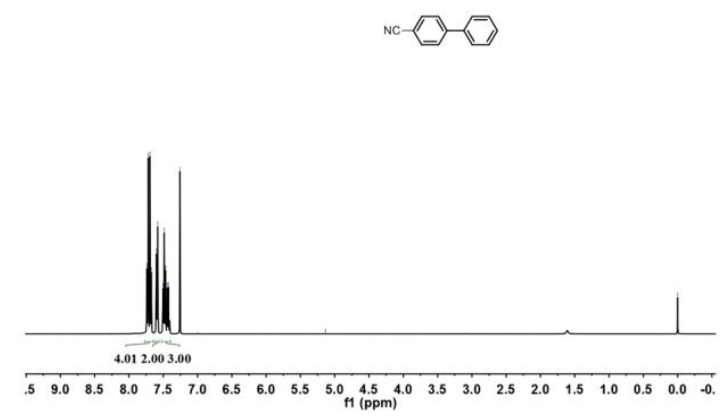

Fig. S19. ${ }^{1} \mathrm{H}$ NMR of the coupling products in $\mathrm{CDCl}_{3}$ at $400 \mathrm{MHz}$. 